\title{
Optimización y comparación entre la micro-extracción en fase líquida y la micro-extracción en fase sólida para la determinación de isoftalaldehido en muestras de migración acuosa.
}

\author{
Jazmín Osorio, Sara Úbeda, Margarita Aznar, Cristina Nerín* \\ Grupo Universitario de InvestigaciónAnalítica (GUIA) \\ Instituto de Investigación en Ingeniería de Aragón (I3A) \\ Universidad de Zaragoza, Mariano Esquillor s/n, 50018, Zaragoza, España. \\ Tel.+34-976761873, e-mail: jazmin.osorio@udea.edu.co
}

\section{Resumen}

En este trabajo, fueron evaluadas dos técnicas de pre-concentración: micro-extracción en fase líquida con fibra hueca (HF-LPME) y microextracción en fase sólida (SPME) para el análisis de isoftalaldehido por cromatografía gaseosa acoplada a espectrometría de masas (GC-MS) en migraciones acuosas de PET, material de envase alimentario. Por normativa, este compuesto debe estar por debajo de $10 \mathrm{ng} / \mathrm{g}$.

\section{Introducción}

El isoftalaldehido es un producto de oxidación del PET, uno de los polímeros más utilizados en la producción de envases alimentarios. Al ser un compuesto no añadido intencionalmente (NIAS) es impórtate controlarlo ya que la legislación EU/10/2011, que regula el uso de materiales en contacto alimentario, no permite superar $10 \mathrm{ng} / \mathrm{g}$ en migraciones. La GC-MS es la técnica más apropiada para el análisis del isoftalaldehido, sin embargo no es posible llegar a los niveles de detección requeridos, por ello surge la necesidad de buscar técnicas de preparación de muestra que nos permitan pre-concentrar. La HF-LPME y la SPME tienen factores de concentración muy altos permitiendo mejorar la sensibilidad para determinados analitos[1]. En este trabajo se comparan dichas técnicas y se analizan muestras de migración con dos simulantes acuosos diferentes.

\section{Materiales y métodos}

\section{Materiales de envase y estudios de migración}

Botellas de tereftalato de polietileno (PET), suministradas por empresa de alimentos.Las botellas se llenaron con dos simulantes acuosos (etanol $10 \%$ y acido acético $3 \%$ ) y se mantuvieron a $40^{\circ} \mathrm{C}$ durante 20,40 y 80 días.

\section{Técnicas de pre-concentración}

HF-LPME: El parámetro mas impórtate en esta técnica es el solvente orgánico, por ello se evaluaron seis solventes: dicloromentano, $\mathrm{p}$ xileno, tolueno, metil-tert-butileter, éter dietilico y benceno. Estas fueron llevadas a cabo con una bomba programable multi-jeringa marca Aladdin AL-8000. Además se evaluaron temperaturas de extracción de 25 y $50^{\circ} \mathrm{C}$, velocidades de extracción de $2.5,5$ y $10 \mu \mathrm{l} / \mathrm{min}$, y volúmenes de extracción de 50,70 y $100 \mu 1$.

SPME: Se evaluaron tres parámetros en el proceso: la naturaleza química de la fibra, el tiempo de extracción y la temperatura de extracción. Este análisis se realizó por inmersión total de la fibra utilizando un auto-muestreador CombiPAL. Las fibras evaluadas fueron PDMS $\left(\begin{array}{ll}100 & \mu \mathrm{m}\end{array}\right), \quad$ PDMS/DVB $(65 \mu \mathrm{m}) \quad \mathrm{y}$ DVB/PDMS/CAR $(50 / 30 \mu \mathrm{m})$. Posteriormente se estudiaron tiempos de extracción de 5, 15 y $15 \mathrm{~min}$ y temperaturas de extracción de 60,80 y $100^{\circ} \mathrm{C}$. En todos los casos solo se modificó el parámetro en estudio manteniendo las demás condiciones constantes. Se tomó el área como el criterio de selección para condiciones óptimas en ambas técnicas.

\section{Instrumentación y condiciones de análisis}

GC-MS: El análisis se llevó a cabo en un cromatógrafo de gases GC $7890 \mathrm{~N}$ acoplado a un detector de masas 5977D (Agilent) y en modo SIM siguiendo los iones 105 y 133 . Con una columna HP-5MS $(30 \mathrm{~m} \times 0.25 \mathrm{~mm}$ diámetro $\times 0.25 \mu \mathrm{m}$ espesor interno) y con una rampa de calentamiento 
$50^{\circ} \mathrm{C} \quad(5 \mathrm{~min}) / / 10^{\circ} \mathrm{C} / \mathrm{min} / / 300^{\circ} \mathrm{C}(5 \mathrm{~min})$. La cuantificaciónen los ensayos de migración se realizó por medio de una curva de calibrado con el estándar puro y siguiendo la misma metodología para el análisis de las muestras.

\section{Resultados}

\section{Optimización}

En la técnica HF-LPME el solvente más adecuado para la extracción fue el tolueno debido a su polaridad, similar a la del analito, tuvo una mayor interacción con el .Adicionalmente, el tolueno no expandió la fibra hueca, como en el caso del diclorometano y el metil-t-butileter, impidiendo la contaminación de las muestras. La velocidad de extracción seleccionada fue de $2,5 \mu \mathrm{l} / \mathrm{min} \mathrm{y}$ el volumen de $70 \mu \mathrm{l}$ porque a estas condiciones se favoreció la migración del analito a través de la fibra $\mathrm{y}$ posteriormente $\mathrm{su}$ interacción $\mathrm{La}$ temperatura óptima seleccionada fue de $50^{\circ} \mathrm{C}$ [2].

En la SPME, la fibra de CAR/DVB/PDMS obtuvo una mayor eficiencia en la extracción. El tiempo de extracción óptimo fue de $15 \mathrm{~min}$, tiempo en el que se alcanzó el equilibrio. Finalmente la temperatura de extracción óptima fue de $80^{\circ} \mathrm{C}$.

\section{Comparación entre HF-LPME y SPME}

En los resultados de la tabla 1 se observan los resultados analíticos de ambas técnicas. En ambos casos se obtuvieron valores satisfactorios de $\mathrm{R}^{2}$. Sin embargo los límites de detección (LOD) y cuantificación (LOQ) fueron más bajos en la HFLPME, este resultado indica que esta técnica tiene mayor sensibilidad para el análisis en comparación con la SPME.

Tabla 1. Resultados analíticos de HF-LPME y la SPME en el análisis del isoftalaldehido.

\begin{tabular}{|l|c|c|}
\hline Parámetro & HF-LPME & SPME \\
\hline LR (ng/g) & $30-540$ & $700-5000$ \\
\hline $\mathrm{R}^{2}$ & 0,988 & 0,978 \\
\hline LOD (ng/g) & 10 & 250 \\
\hline LOQ (ng/g) & 30 & 700 \\
\hline
\end{tabular}

\section{Cuantificación en muestras de PET}

Los resultados de las muestras migración con dos simulantes diferentes, etanol $10 \%$ y acido acético $3 \%$, se pueden apreciar en la gráfica 1 . Se observó que la concentración de isoftalaldehido en ambos simulantes era similar y que la concentración del compuesto en ambos casos aumentaba en el tiempo.

\section{Conclusiones}

En este estudio se encuentra que ambas técnicas son idóneas para la cuantificación de isoftalaldehido en muestras de migración acuosa de botellas de PET. La SPME es más conveniente para la cuantificación a concentraciones más altas, sin embargo la HF-LPME es la técnica que se ha implementado debido a que permite alcanzar el límite de detección exigido por la legislación.

\section{REFERENCIAS}

[1]. OLIVEIRA, É. C., Y. ECHEGOYEN, S. A. CRUZ AND C. NERIN Comparison between solid phase microextraction (SPME) and hollow fiber liquid phase microextraction (HFLPME) for determination of extractables from post-consumer recycled PET into food simulants. Talanta, 2014, 127, 59-67.

[2]. PEZO, D., J. SALAFRANCA AND C. NERÍN Development of an automatic multiple dynamic hollow fibre liquid-phase microextraction procedure for specific migration analysis of new active food packagings containing essential oils. Journal of Chromatography A, 2007, 1174(1-2), 85-94

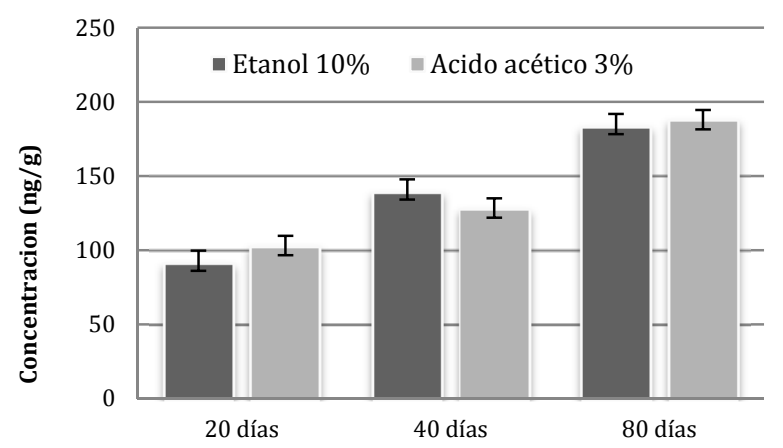

Grafico1. Concentración del isoftalaldehido en muestras migración acuosas a 20, 40 y 80 días. 